\section{ECONOMÍA, SOCIEDAD Y ÉTICA: UNA PROPUESTA INTEGRATIVA}

\author{
José Atilano Pena López \\ Departamento de Economía Aplicada I \\ Facultad de Economía y Empresa \\ atilano@udc.es
}

\section{ECONOMICS, SOCIETY AND ETHICS: AN INTEGRATIVE PROPOSAL}

\begin{abstract}
This present work starts from a critical review of the diverse approaches that attempt to locate the economic fact within the social system and the perspective that Ethics adopts on the economy. On these grounds, the reconstruction of the Das Adam Smith Problem allows us to establish the foundations of an integrating socioeconomic rationality. At the same time, the proposal of an integrative ethics that surpasses the traditional dichotomies between facts and values and the split between economic and social rationality, would allow us to overcome the errors that ethical analysis makes in its judgments on the internal logic of Economics.
\end{abstract}

KEY WORDS: Sociology of economics, Integrative Ethics and economy, Das A. Smith Problem.

\section{INTRODUCCIÓN}

En la actualidad es un lugar común en Economía destacar la influencia de las reglas, hábitos, normas e instituciones en general en las prácticas económicas. Los procesos económicos se encuentran integrados en los sociales, de forma que no existe un capitalismo establecido, sino sólo diferentes variedades caracterizadas por un legado cultural, unas bases normativas y una determinada configuración social. Igualmente, si nos centramos en el ámbito de la ética económica, resulta obvio el hecho de que los mercados y los fenómenos económicos asociados dependen y al mismo tiempo influencian los "sentimientos éticos o morales", las normas y los comportamientos.

No obstante, pese a esa interrelación, se admite la oposición entre ética y economía, como si la primera de ambas fuese la expresión de lo que precisa ser corregido en la segunda. Es evidente que en términos analíticos es posible distinguir el proceso de elección sobre la asignación de recursos de la determinación de las preferencias, sin embargo, en la práctica de los individuos se mezclan de tal forma que estos deciden simultáneamente sobre ambas de un modo integrado (Koslowski, 1991).
RESUMEN: El presente trabajo propone una revisión critica de los diversos enfoques que proponen situar el hecho económico dentro del social, así como de las perspectivas que la Ética adopta sobre la economía. Partiendo de este análisis, el replanteamiento del Das Adam Smith Problem permite sentar las bases de una racionalidad socioeconómica integradora. Ésta propuesta de una ética y economía integrativas supera las dicotomías tradicionales entre hechos y valores y la escisión entre racionalidad económica y social, asi como, los errores que el análisis ético comete en sus juicios sobre la lógica interna de la Ciencia Económica.

PALABRAS CLAVE: Sociología económica, Ética económica integrativa, Das A. Smith Problem.

Un ejemplo clásico en este sentido es el conocido trabajo de R. Titmus (1971) sobre los mercados de sangre frente las donaciones de sangre. Este autor comprobaba como la aparición de un mercado de sangre minaba el compromiso de donación que previamente caracterizaba a los agentes. En sentido inverso, las convicciones morales pueden verse influenciadas causalmente por la Teoría Económica, de hecho, como ha sido crecientemente comprobado a partir del experimento de Marwell (1981), una mejor preparación en Elección Racional acaba por justificar personalmente los comportamientos autointeresados.

Esta dicotomía entre lo ético y lo económico no constituye un hecho aislado sino que forma parte de un problema compartido por las ciencias sociales. Se trata, por ejemplo, del mismo que surge al ahondar en la relación entre ética y política. Ahora bien, no cabe pensar en la ética como un sustitutivo de la economía o, en su caso, de la política.

En el presente trabajo nos proponemos revisar de forma bidireccional el problema de los dualismos presentes en el estudio de la interrelación entre sociedad y economía y sus derivaciones en los existentes sobre la interrelación entre ética y economía. Así, en el segundo apartado haremos un 
breve recorrido sobre las propuestas de integración de los hechos económicos en la realidad sociocultural para, seguidamente, considerar las derivaciones que este problema tiene sobre los enfoques o perspectivas de la moral sobre la economía. El cuarto apartado introduce la perspectiva smithiana como una base sobre la que asentar una visión integrativa que supera las disociaciones de las propuestas precedentes. En este sentido, tal y como veremos, la obra de A. Smith es particularmente relevante, en la medida en que su teoría económica se encuentra profundamente arraigada en una concepción previa del orden moral. Finalmente, se plantea una propuesta integrativa, para la que la comprensión de la filosofía moral permite enriquecer las bases del análisis económico.

\section{LO ECONÓMICO FRENTE A LO SOCIOCULTURAL}

La Economía en su versión neoclásica' se limita a abordar la lógica funcional del sistema de mercado y se presenta a sí misma como una explicación pura de las interacciones entre los individuos, neutral en términos de valores, modelada a través de unos determinados presupuestos antropológicos a los que comúnmente nos referimos como homo oeconomicus (Hausman y McPherson, 2008). Para el individualismo metodológico, característico de esta visión, los agentes no precisan conocer las dimensiones éticas de las interacciones humanas, puesto que la teoría defiende un ideal de sociedad que funciona como un sistema de "egoísmo" autoordenado.

Ahora bien, el objeto propio de la Economía es una compleja práctica social en la que se ven implicados valores, normas e instituciones, es decir, no puede entenderse como libre de contexto o neutral desde un punto de vista valorativo. Consecuentemente, es necesario ahondar en el modo en que lo social y lo ético se incorpora en el análisis de los procesos económicos para lo cual realizaremos un breve recorrido sobre los conceptos utilizados para describir esta relación.

\subsection{Embedded, Enmeshed, framed}

Un mercado puede ser definido en su sentido más literal como un conjunto de instituciones sociales en el cual se realizan intercambios regulares. Expresado de otro modo, es un producto de unas instituciones previas sobre las que se asienta (Hodgson, 1989). Es más, no es el modo único de coordinación de la división del trabajo, sino que es dependiente de otras formas de coordinación de no-mercado que constituyen su substrato. En realidad, el argumento comúnmente abordado de que las fuerzas del mercado adquieren autonomía autorreguladora al margen de las intenciones y acciones de los agentes carece de entidad y acaba derivando en una reacción defensiva, frente a sus supuestos efectos destructivos. En último término, las fuerzas del mercado son sociales en el más amplio sentido de la palabra y tras toda valoración económica subsiste una valoración moral (Anderson, 1993; Sayer, 2004).

El ser humano en cuanto ser social no puede establecer relaciones entre individuos sin adoptar decisiones morales, aun cuando buena parte de ellas sean realizadas tácita o "inconscientemente" en lo que denominamos disposiciones éticas o habitus. En la práctica, los comportamientos se basan en una mezcla de convenciones que van desde el interés particular hasta el compromiso y las normas morales.

En definitiva, sin prestar atención a la dimensión moral no es abordable ninguna forma de relación social y, más en concreto, las relaciones económicas también se construyen de normas sobre derechos, responsabilidades y comportamientos apropiados. Pero, al mismo tiempo, abordar la moral como un conjunto de normas y reglas respaldadas por sanciones que tratan de generar un orden social es generar una concepción alienada de esta dimensión de la vida social. El hombre no sólo se comporta de un determinado modo por el miedo a un castigo sino porque cree que es correcto o incluso porque cree que ello puede beneficiar a otros.

Los estudios sobre el encuadramiento de los hechos económicos en la realidad social han sido dominados por los trabajos clásicos de Polanyi (1944) ${ }^{2}$ y por el concepto de embedment. Ahora bien, conviene situar este enfoque para valorar críticamente sus aportaciones. Cuando este autor introdujo el término embedded ${ }^{\beta}$ (integrado) lo hace para aludir a las transformaciones que había supuesto la revolución industrial (disembedding) respecto a las sociedades tradicionales. Este cambio, para Polanyi, dio lugar a una inversión de prioridades en la que las relaciones económicas pasaron de estar integradas en las sociales a 
generar lo que denominamos una "sociedad de mercado" en la que existe un dominio de lo económico sobre las restantes expresiones sociales. Esta afirmación debe ser puntualizada. La economía cultural ha puesto de manifiesto que lo que Polanyi consideraba una forma de ruptura no era más que una reconfiguración de las relaciones dominantes. Los procesos económicos pueden romper sus vínculos con ciertas expresiones sociales (identidades étnicas, lazos familiares...) para apoyarse sobre otra forma de regulación social que "estabiliza las posibilidades de cálculo económico y las conductas económicas" (Jessop, 2000). La apariencia que se genera es que la sociedad es un adjunto del mercado, pese a que, tal y como recalca Granovetter (1985), los procesos capitalistas contemporáneos continúan enmeshed, (capturados, entrelazados 0 enredados) en el entramado social bajo la percepción de que se trata de una vinculación externa.

Ambas nociones clásicas, embedded y enmeshed, no pueden escaparse de una concepción de las instituciones sociales y éticas como imposición externa y no una precondición al funcionamiento de la propia economía. En cierta medida, incluso cabría una lectura simplista en la que la sociedad se limita a redes de individuos ajenas al mercado, cuando la realidad es que los procesos económicos y sociales se mezclan de una forma que supera las barreras establecidas arbitrariamente por ambas disciplinas (Kripper, 2001). En particular, el establecimiento de los derechos de propiedad, del derecho en general, la fijación de las responsabilidades y las reglas del juego, son precondiciones necesarias para una acción económica ordenada. Lo que es más, cuanto mayor sea la complejidad de la actividad económica o, lo que es lo mismo, a mayor división del trabajo, más importante es la resolución de estos problemas.

En una línea semejante, se encuentra el concepto de estructuración social y moral (framing) de la acción económica planteado por Callon (1998). Toda actividad se soporta sobre la exclusión de ciertas posibilidades y bajo unos presupuestos y normas compartidos por los agentes, es decir, un marco moral. Esto es particularmente evidente en el mercado si tenemos en cuenta que su principal apoyo es la existencia de una serie de presupuestos sobre transacciones y responsabilidades implícitas. Ahora bien, si tenemos en cuenta como se mezclan las motivaciones en las actuaciones individuales, resulta imposible realizar diferenciaciones categóricas. Esta es la dificultad que en- contramos para escindir lo económico de lo cultural o lo moral. Es posible hablar del carácter estructurante de lo cultural o moral sobre la acción económica, pero la realidad desborda una concepción de estructura o influencia externa.

En todas estas interpretaciones, el problema no reside en reconocer la presencia de influencias externas al mercado, por otra parte evidentes, sino en no considerar que los valores y normas son internos a las propias prácticas económicas en sí mismas.

\subsection{La integración del hecho económico en el entramado sociocultural}

A partir de lo señalado, situar el hecho económico dentro del entramado social implica tener en cuenta que se trata de una acción social, no ajena al entramado social en el que se sitúa y regida por instituciones sociales. Tres orientaciones han tratado de proponer alguna forma de integración: la sociología de la economía, la teoría del capital social y la economía neoinstitucional.

\section{La acción económica es una acción social: la sociología de la economía}

Si aceptamos la definición tradicional de la acción económica como la elección entre diversos usos de recursos escasos, el comportamiento económico es esencialmente maximizador y racional y las influencias sociales podrían ser consideradas un "ruido perturbador". El estudio de la economía en la mainstream ${ }^{4}$ neoclásica avanzó focalizado en el análisis de la toma de decisiones en búsqueda de la eficiencia. Sin embargo, tal y como hemos señalado, desde el punto de vista sociológico, es obvio que la acción económica no puede separarse de la búsqueda de aprobación, estatus, sociabilidad y poder.

El cómo se produce esta influencia es uno de los problemas esenciales a los que las distintas escuelas en el ámbito de la sociología del hecho económico han tratado de dar respuesta ${ }^{5}$. A título ilustrativo, dos hitos clave en el nacimiento de la Sociología ya hacian hincapié en este problema. Durkheim (1893) enfatizó como la acción económica pura fracasa en vincular individuos más allá de instantes de intercambio puntual. Weber (1994 [1905]), a su vez, puso de manifiesto como el comportamiento económico de los 
individuos no puede analizarse de forma aislada al de los otros. Cada sujeto toma en consideración los comportamientos de los demás a través de significados construidos socialmente. Burawoy (1985), por ejemplo, evidenció la importancia de la competencia y emulación en la productividad de los trabajadores integrados en un grupo.

En último término, las actuaciones económicas están inspiradas por representaciones colectivas entre las que se encuentran convenciones tales como el "valor monetario". Por otra parte, la acción económica no es comprensible si prescindimos del hecho de que se trata de la mayor fuente de poder en la sociedad.

\section{La acción económica está situada socialmente: la teoría del capital social}

Las acciones de los individuos no sólo no pueden ser explicadas atendiendo exclusivamente a motivaciones individuales, sino que las conductas se sitúan en redes de relaciones.

El concepto de "red" es el eje de la teoría del capital social ${ }^{6}$. Su utilidad en el análisis sociológico de la economía radica en la proximidad a la realidad empirica concreta. En conjunto, al aludir al término red se pone de relieve la influencia de la interacción entre individuos y grupos para explicar los comportamientos económicos. Las referencias podrian ser interminables ya que estamos ante una evidencia de la vida diaria. El modo en que un individuo logra un empleo o planifica una inversión se explica mejor atendiendo a la red social en que se integra que a los mecanismos puros de mercado. Recurriendo a los trabajos más clásicos sobre el tema, multitud de estudios ponen de relieve como las minorías étnicas $y$, en particular, sus redes, presentan ciertas facilidades para algunas actividades (financieras, productivas...) (Curtin, 1984). Negocios tan complejos como el bancario manifiestan una clara tendencia a funcionar en red, estableciendo vínculos estables con una clientela cuasi-fija asociada a alguna forma de relación personal. Igualmente, en lo que respecta a la fijación de precios, Sahlins (1974) observaba rigideces en su modificación asociadas a vínculos personales. En esta misma línea, Okun (1981) llegó a plantear la existencia de dos tipos de precios: Precios de opción-subasta y precios de mercadoconsumidor. En tanto que los primeros se adaptan a un mercado clásico de oferta y demanda despersonalizado y sólo serían aplicables a productos homogéneos, en los segundos, en cambio, existe una influencia de la relación entre oferente y demandante.

En suma, determinar en qué medida son más influyentes las fuerzas sociales o las de la "libre" interacción de oferta y demanda, es un caso de estudio en cada mercado particular. Lo social y lo económico se mezclan de un modo difícilmente escindible

\section{El carácter social de las instituciones económicas: el neoinstitucionalismo}

La Escuela Neoclásica, a la que venimos aludiendo como mainstream, concibe un mundo "sin fricciones", en el que los intercambios tienen lugar en mercados perfectamente competitivos ajenos a la existencia de costes de adquisición de información, incertidumbre y costes de transacción. Los estudios del Neoinstitucionalismo, en cambio, presentan como núcleo común la creencia de que la Ciencia Económica debe abordar el estudio de las instituciones sociales (Williamson, 1981 y 2000). Sin embargo, no proponen un paradigma alternativo, sino sólo complementario. El argumento empleado como justificación de la existencia y permanencia de las instituciones es su capacidad para economizar los costes de transacción, es decir, sólo su funcionalidad económica.

Instituciones, como la moral, son modos de resolución de problemas sociales de coordinación, que evitan que las acciones individuales desemboquen en resultados colectivos socialmente indeseables. Dicho de otro modo, son modos de articular los intereses individuales. Los grupos humanos afrontan problemas evolutivos cuya solución queda plasmada en el establecimiento de una institución

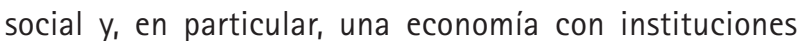
eficientes dispone una de las claves del crecimiento (North, 1990).

Dentro de este tipo de trabajos merece la pena destacar las aportaciones de R. Coase. Este autor en un artículo de 1937 justificaba las nociones de empresa y empresarialidad como una institución social ligada a la necesidad de ahorrar costes de agencia (vigilancia, información...) mediante la creación de vínculos contractuales estables entre individuos. 
Pese al éxito de esta aproximación, es preciso reconocer que el concepto de eficiencia en economía resulta notablemente contradictorio y falto de "sutileza" (Obershall y Leifer, 1986). Abundando en esta idea, la construcción social de la realidad planteada en las teorias de Berger y Luckmann (1966), también cuestiona que las instituciones sean lo que externamente aparecen. En general, es imposible comprender una institución sin entender el proceso que condujo a su establecimiento. No obstante, la aparente limpieza argumental de esta propuesta no puede conducirnos al simplismo de que son siempre las instituciones más eficientes las que perviven. El estudio de las situaciones QWERTY ${ }^{7}$ en las que perviven las soluciones menos eficientes pone de relieve las imperfecciones del sistema.

\section{El problema del anÁlisis Ético de la ECONOMía}

Tras esta breve revisión podemos hacer una lectura en sentido inverso. Las deficiencias de integración del marco social en el análisis de hecho económico tienen también su traducción en la perspectiva que la Ética asume en su análisis de la realidad económica y de la Economía en cuanto ciencia. Sintéticamente podrian distinguirse dos modos de comprensión de esta relación en razón de cuál de las dos asume el papel predominante: Correctiva y funcionalista.

La ética correctiva propone un uso de la racionalidad ética externo a la propia economía y que por lo general deriva en condenas directas de la lógica del mercado. Esta orientación plantea una confrontación directa con la economía convencional en la que la ética se convierte en un guardián externo de la economía, condenando de modo taxativo el funcionamiento del mercado, por considerarlo fuera del área de actuación del altruismo. De hecho, en último término, se estaría estableciendo una identidad entre este último y la ética ${ }^{8}$.

Esta actitud de conflicto puede considerarse directamente ligada a concepciones pre-modernas del funcionamiento de la economía. En una sociedad en la que el juego dominante fuese de suma cero ${ }^{9}$, esto es, en la que la mejora de la posición de unos fuese ligada al deterioro de la de otros, tendería a plantearse una ética que corrigiese los resultados del mercado. De hecho, la prohibición canónica del préstamo a interés y la condena a la acumulación de capital, emplean claramente argumentaciones de juegos de suma cero. Las sociedades modernas, en cambio, que se caracterizan por estar integradas por múltiples sistemas funcionales y una tendencia al crecimiento, se ajustan más a modelos de suma positiva, en los que cabe la mejora de posición de ambos jugadores. Bajo estas circunstancias es compatible la búsqueda del interés personal con valores como la solidaridad y se desvirtúan las propuestas éticas correctivas, dando mayor protagonismo a las de corte funcionalista (Luetge, 2005).

El enfoque funcionalista puede considerarse la tendencia dominante en al actualidad, en especial en el ámbito anglosajón. Para éste, la ética favorece las relaciones de mercado y es un componente funcional del sistema.

Como elemento común de las diversas orientaciones dentro de esta perspectiva está la percepción de la existencia de una demanda de ética en las economías de mercado. Por una parte está el análisis ético-culturalista de herencia weberiana al que subyace la recuperación de la ética fundacional del capitalismo y, por otra, algunos planteamientos que consideran la ética de empresa como un modelo de gestión garante de una mayor eficiencia.

Para el análisis ético-culturalista, el sistema ético-cultural es la principal fuente de dinamismo que explica el acceso a un sistema político democrático y de economía liberal. Éste es propiamente una infraestructura, una "red de ideas y sentimientos" sobre la que se asienta tanto el subsistema político como el económico. Tal y como señala Novak (1988), un sistema económico siempre se apoya sobre un sentido del deber, de la disciplina, de la creatividad y del ahorro y los problemas de ausencia de una identidad ética se traducen en fallos del subsistema económico en su conjunto. Incluso una crisis del sistema sería una consecuencia directa de sus propias contradicciones internas, en especial las éticas, dado que, de un lado tiene como fundamento una ética típicamente puritana pero, simultáneamente, precisa la existencia de una ética hedonista para el consumo (Martínez Real, 1998) ${ }^{10}$.

Por tanto, esta perspectiva sostiene, partiendo del consecuencialismo utilitarista, una ética funcionalmente necesaria; idea a la que se adscriben en cierta medida también otras orientaciones, como es el caso de la Escuela Austriaca. 
Así, por ejemplo, Hayek afirma que "un sistema moral debe producir un orden funcional, capaz de preservar el aparato de civilización" (Hayek, 1994, p. 118), ya que la moral es la base de la cooperación en la que se apoya todo el funcionamiento económico. Expresado de otro modo, cuanto ampara la cooperación debe considerarse moral y cuanto va en detrimento de ella, inmoral (Mises, 1994, 1995).

No obstante, bajo ese epígrafe general de funcionalismo es preciso distinguir diversos enfoques que van desde el contractualismo constitucionalista al republicanismo, entremezclando dimensiones correctivas y funcionalistas.

El constitucionalismo de Buchanan trata de poner de manifiesto el valor económico de las normas éticas, en particular de la ética del trabajo y del ahorro. En último término, este autor destaca la productividad de los códigos éticos de comportamiento para fundamentar una ética procedimental del orden constitucional (Buchanan, 1991). La ética se configura así, como un conjunto de reglas abstractas que fijan los límites de las estructuras institucionales, es decir, delimita un espacio de posibilidades que facilita el ordenamiento económico. Esta perspectiva es próxima al institucionalismo, en la medida en que se centra en el estudio del conglomerado de reglas e instituciones que dan forma a la interacción humana facilitando la reducción de los costes de transacción (North, 1990 y Sugden, 1986). La Socioeconomía, en cambio, trata de conjugar de una forma más o menos equilibrada las perspectivas deontológicas y consecuencialistas, al admitir una doble dimensión en las preferencias: el yo y el nosotros (Etzioni, 1988). Los seres humanos constituyen para esta orientación simultáneamente individuos y miembros de una comunidad, con lo que la ética penetra los procesos de decisión "racionales" de los agentes al incorporar el "nosotros" del grupo en el que se integra el individuo. Sin embargo es dualista, pese a que trata de integrar ética y racionalidad económica, ambas constituyen dos compartimentos estancos ${ }^{11}$ y la ética desempeña una función limitativa o correctiva. Esta función correctiva todavía se hace más evidente en el republicanismo económico y en el funcionalismo de Homman. Para el primero, el concepto de ciudadanía económica constituye un a priori que debe garantizarse. La ética, en este caso, es un criterio para diferenciar los modos de ejercicio del poder en orden a favorecer la libertad de los ciudadanos (Cortina y Conill, 2001; Conill, 2004). Para el segundo, la racionalidad económica marca la pauta de lo moralmente realizable en la complejidad de la vida económica. Por tanto, disuelve la aparente contradicción entre moral y economía aprovechando las condiciones de realización de ésta en la vida económica (Homann y Pies, 1994).

Tal y como señalábamos anteriormente, dentro del funcionalismo, junto al análisis ético-culturalista, se encuentra la denominada Ética de los Negocios o Ética de Empresa (Business Ethics). A este enfoque le subyace un sistema de gestión empresarial centrado en la cooperación, con el objetivo de reducir los costes y facilitar el desarrollo de tareas comunes. La cooperación puede verse obstaculizada por la carencia de una cultura empresarial de respeto a las relaciones contractuales, de ahí la importancia del establecimiento de relaciones de confianza y lealtad inter e intraempresariales. La introducción de la ética se justifica en razón del propio funcionamiento empresarial y de la búsqueda de un beneficio de largo plazo. En el mejor de los casos, tal y como señala A. Cortina, "una ética empresarial no es una ética de la convicción, sino una ética de la responsabilidad por las consecuencias de las decisiones que en ella se toman" (Cortina, 1992) ${ }^{12}$. En cierta medida, esta orientación podría ser considerada un análisis económico de la ética, pero no un ejercicio de la actividad ética crítica.

\section{LA PROPUESTA SMITHIANA}

Una comprensión de la actividad económica imbricada en unas precondiciones normativas permitiría una aproximación más fluida al papel de la ética en la economía. El replanteamiento actual del denominado "problema smithiano" puede resultar esclarecedor en la construcción de una ética económica integrativa. Se trata de una controversia, de larga tradición en ética, centrada en la contradicción aparente entre las principales afirmaciones de este autor en su obra moral ("La teoría de los sentimientos morales") y su obra económica ("La riqueza de las naciones"). A título de ejemplo y recogiendo las dos citas más celebradas de ambas obras, parece existir un conflicto. En el comienzo de primera puede leerse:

"Por más egoista que pueda suponerse un hombre, existen evidentemente en su naturaleza algunos principios que le 
hacen interesarse por la suerte de los otros, y que hacen que la felicidad de estos resulte necesaria, aunque no se derive de ella más que el placer de contemplarla" (TSM, I, 1).

En tanto que en el fragmento más citado de La Riqueza de las Naciones se afirma:

"No de la benevolencia del carnicero, del cervecero o del panadero es de quien esperamos nuestra cena (...) sino de su interés particular" (RN, I, 2).

La primera parece afirmar la existencia de una tendencia natural a la prosocialidad en el hombre, que podría entrar en contradicción con el carácter esencial que se concede al interés particular en la segunda. No obstante, para los estudios más recientes sobre el Das A. Smith Problem ${ }^{13}$ (Pena y Santos, 2007), el pensamiento smithiano constituye un todo perfectamente articulado entre moral y economía. Ambas obras parten de lo podríamos denominar como "psicología moral", un análisis de las motivaciones determinantes en el actuar que abarca aspectos que en la actualidad serían objeto de Sociología, Economía o Ciencia Política. El concepto clásico de sentimientos morales presente en la obra de Smith hace referencia al hecho de que una acción no se encuentra exclusivamente influenciada por el interés particular, sino igualmente por los juicios morales y las decisiones de los individuos respecto a otros y a sí mismos y las instituciones sociales. Entre ellos, Smith presta una atención particular a la simpatía, es decir, como el bienestar de los individuos se ve afectado por la situación de los otros.

Un breve recorrido sobre las diversas vertientes hermenéuticas de la obra smithiana puede ser paradigmático a la hora de comprender los problemas de la interrelación entre Ética y Economía.

A efectos de síntesis y sistematización, los intentos de integración de la obra smithiana pueden clasificarse estableciendo una triple división. En primer lugar, algunos autores interpretan la moral construida sobre las relaciones de simpatía como el marco limitativo o las reglas básicas de juego del interés particular (Anspach, 1972; Wilson, 1975; West, 1976). Mediante la simpatía las sociedades construyen una moral que corrige los excesos del interés individual. La moral tendría entonces un carácter exógeno con respecto a las propias relaciones económicas y el problema de Smith tendría toda su entidad.
En segundo lugar, existen interpretaciones que entienden la simpatía como un vínculo que delimita un entorno de proximidad para cada individuo. Una esfera en la cual no existen relaciones de interés particular, sino de interdependencia o comunidad; lo que supondría aceptar que la moral quedase relegada a los ámbitos de no-mercado (Nieli, 1986). La simpatía delimita, por tanto, donde no entra el interés individual, con lo que la moral y el altruismo serían ajenos al mercado.

Finalmente, está la postura que considera la simpatía como un vínculo común y universal presente con diversos grados en cualquier modo de relación y con efectos en la reducción de los costes de transacción. Bajo esta última perspectiva en la actuación individual operan simultáneamente tanto el interés particular como los vínculos de simpatía. Esta última interpretación deja cauces abiertos para incorporar aspectos de la socialidad que tradicionalmente no han sido abordados al analizar los comportamientos económicos. De la noción de simpatía se pueden derivar importantes implicaciones adicionales, como por ejemplo, la aparición de sentimientos de pertenencia, identidad 0 comunidad (Sugden, 2002; Anderson, 2001) ${ }^{14}$, que difícilmente podrían ser incorporados a los modelos formales al uso en el marco de la Elección Racional ${ }^{15}$. La vida social humana se articula a través del intercambio de sentimientos de correspondencia, que pueden acabar por configurar formas de razonamiento colectivo en los que los individuos operan como si se tratase de un único agente, sujeto plural o pensamiento de equipo (we rationality).

Lo que es más, para Smith los individuos no sólo son económicamente dependientes en el proceso de división del trabajo, sino que igualmente son psicológicamente dependientes. Mediante la simpatía, la situación de los otros repercute en el proceso de toma de decisiones por parte del agente y sus acciones son juzgadas por un "espectador imparcial" constituido por un proceso de socialización. Los seres humanos precisan de compañía y reconocimiento y por tanto se encuentran vinculados y afectados por la situación de los otros. Incluso llega a considerar que los sujetos evalúan su conducta imaginándose sus efectos y los juicios que un espectador imparcial realizaría sobre los mismos. Smith está así considerando el papel de las emociones en la moral de un modo cognitivo, lo cual lo aproxima a propuestas como las de M. Nussbaum (2001) para la que las emociones son abordadas como juicios 
evaluativos en relación a asuntos que afectan al bienestar de los individuos.

Adicionalmente, la simpatía contribuye a la búsqueda de acuerdos que sean mutuamente benéficos y potencia la división del trabajo. Es decir, los intercambios se asientan en la capacidad comunicativa del hombre, capacidad ésta que se erige como base del sistema social y del progreso económico. La moral constituye, consecuentemente, un sistema de precoordinación o una base de socialidad elemental sobre la que se sustenta la coordinación del mercado $^{16}$ (Young, 1997).

La aludida noción smithiana de simpatía permite, por un lado, corregir el paradigma tradicional en Economía $y_{\text {, }}$ por otro, imbricar tanto la ética y los comportamientos prosociales con el interés particular en el proceso de toma de decisiones. Consiguientemente, el sistema económico condiciona la moral $y$, a su vez, la moral es determinante del funcionamiento del sistema económico.

Los comportamientos económicos, incluso aquellos más aparentemente amorales se encuentran afectados por las normas sobre como deben ser tratados los otros, pero no interpretables en términos de mera coacción externa o marco de ejercicio de la actividad, sino como un proceso complejo de toma de decisiones en los que se entremezclan aspectos culturales, morales y psicológicos, y donde el interés particular no es el único principio de toma de decisiones. Probablemente esta sea la concepción más real de la imbricación, una mezcla fluida de elementos más que una restricción de los procesos económicos.

Mientras que para la Economía Neoclásica la comprensión de los comportamientos cooperativos y la confianza resulta difícilmente modelizable, Smith propone una moral que se adentra en la propia antropología superando los simplismos que la reducen a normas formales respaldadas por sanciones. La evidencia de las relaciones económicas, desde las laborales a las de simple intercambio de mercancías, presupone la existencia de relaciones personales subyacentes y, en éstas, alguna forma de simpatía y compromiso.

En definitiva, la ventaja de la alternativa smithiana reside en que su aproximación ofrece la posibilidad de comprender la moral económica de un modo en el que las consideraciones éticas no están escindidas realmente de las económicas. De esta forma, se elimina la dicotomía tradicional entre hechos y normas y se adopta una racionalidad socioeconómica. Ambos rasgos constituyen los pilares de una economía ética integrativa.

\section{UNA ECONOMÍA ÉtICA INTEGRATIVA}

Una reconstrucción integrativa de estas características podría asentarse sobre dos pilares. Por una parte, sería necesaria una revisión de la dicotomía tradicional entre hecho y norma que permita superar las perspectivas deontológicas y consecuencialistas. Por otra parte, la reconstrucción de una racionalidad holística o socioeconómica.

El problema de la disociación entre Ética y Economía que venimos abordando se encuentra reflejado en la dicotomía entre hechos y valores. La propuesta clásica de Robbins (1932) ponía de relieve la necesidad de esta distinción en orden a establecer discusiones sobre bases objetivas. No obstante, el hecho y los juicios valorativos constituyen un conjunto difícilmente escindible (Putnam, 2002). Así, la idea de dividir los conceptos económicos en una parte descriptiva y otra valorativa es una pura ficción filosófica arraigada en la noción de hecho del positivismo lógico (Hempel, 1963, Quine, 1951). Para esta orientación, la verdad sólo puede asentarse en verdades empíricas o enunciados analíticos en los que se establece una correspondencia directa entre las afirmaciones y la realidad. Ahora bien, en el caso de la economía, la mera descripción de la realidad en términos neutrales no es posible.

Esta misma dicotomía podría trasladarse a la oposición normas-valores que caracteriza la filosofía habermasiana (Habermas, 2000). Las normas informan sobre lo que debe ser, en tanto que los valores sobre qué conducta es deseable, estableciendo prelaciones o preferencias. Nuevamente, se trata de una distinción más metafísica que lógica, ya que una norma es una afirmación de validez universal que implica obligación y los valores presentan un carácter no-cognitivo o incluso subracional. Los supuestos de la racionalidad dialógica habermasiana ponen en cuestión la posibilidad de alcanzar un conjunto de valores intersubjetivos, un único pilar moral, que no puedan ser tildado de etnocéntrico (Putnam, 2003). El único planteamiento uni- 
versal factible es la afirmación de que a la ética le compete el alivio del sufrimiento de los individuos o, expresado en términos positivos, la mejora de su bienestar.

Consecuentemente, las perspectivas deontológicas centradas en la fijación de normas morales universales pueden no constituir un enfoque adecuado por su carácter externo y correctivo. Pese a que es evidente la necesidad de una serie de derechos y normas para garantizar el libre juego del mercado, la deontología se encuentra con el problema de lograr un acuerdo dialógico. Si bien las escuelas heterodoxas (institucionalista, marxista...) serían favorables a la inclusión de normas que permitiesen garantizar supuestas mejoras en los niveles de bienestar, las orientaciones ortodoxas considerarian que éstas afectan negativamente a los incentivos y crearán ineficiencias. Al mismo tiempo, una moral deontológica se encuentra con el problema de su aplicación a situaciones concretas. La vida social es demasiado compleja para ser reducida a un conjunto de derechos y deberes (Walsh, 2003 y Putnam, 2003).

Frente a ésta, la moral de virtudes en el más puro sentido aristotélico presenta una mayor capacidad integrativa. La virtud es adquirida en interacción diaria a través de un sistema de prueba y error. Por tanto, es contextual, centrada en la responsabilidad y trata de alcanzar un equilibrio entre las dimensiones prácticas y evaluativas. Este rasgo sitúa a la ética de virtudes en una posición intermedia entre las éticas consecuencialistas y la preocupación por las normas universales que caracteriza a la moral deontológica (Mclntyre, 1987). Una ética de virtudes reconoce que el bien no presenta una expresión universal y que el comportamiento moral se adapta a las cambiantes circunstancias sociales. En cualquier actuación los agentes toman en consideración no sólo las consecuencias y los deberes, sino que están igualmente sujetos a las circunstancias y, particularmente, a las relaciones sociales en el sentido smithiano al que anteriormente haciamos referencia. En este marco, la práctica económica es definida como un conjunto de actividades capaces de crear valor entre agentes comprometidos en la misma. Esto implica que los agentes están guiados por motivaciones y razones, cuya base sería minada por comportamientos instrumentales. Así, los agentes económicos serian reflexivos y no meramente calculadores, merecedores de confianza y no solamente creibles. En otras palabras, para una ética de virtudes la racionalidad de los agentes debe portar sen- tido y no sólo ser resultado de un cálculo oportunista (Staveren, 2007).

El concepto de capacidades (capabilities) planteado por Sen constituye un excelente ejemplo de imbricación de ambas nociones que supera la crítica de falta de universalidad. Entendemos por capabilities, "capacidades para el ejercicio de funciones valiosas, acciones que un individuo posee razones para valorar" (Sen, 1967) (vida, educación, salud...). Se trata de una noción que va más allá del mero cálculo de la renta incorporando aspectos como las libertades, los derechos, las condiciones de vida, la creatividad... Nos remite, de este modo, a un planteamiento mucho más amplio que integra la ética en la construcción o en la búsqueda de una vida buena (Putnam, 2003).

Del lado de la Economía y en lo que respecta a la construcción de una racionalidad socioeconómica, una ética integrativa supone además una transformación del modo habitual de entender la racionalidad. Una revisión crítica de sus presupuestos axiológicos (Conill, 2004 y Ulrich, 1999).

El "economicismo" que caracteriza a la mainstream neoclásica asume que la moralidad es la racionalidad, lo que conduce a un relativismo y escepticismo ético (Hausman y Mcpherson, 1993). No obstante, la Ética antecede a la Economía dado que el origen de estas valoraciones y motivaciones no es el mercado, aunque éste ejerce una influencia, ni el Estado, a menos que resucitemos la tesis inaceptable de la existencia de un Estado ético, sino la sociedad civil que crea el mercado y el Estado, y le otorga legitimidad (Zamagni, 1989 y 1994). Por otra parte, tal y como ha puesto de manifiesto la economía experimental, la principal característica de los agentes no reside en que estos tengan una deficiente capacidad de cálculo, sino que frecuentemente actúan intuitivamente con lo que su comportamiento tiene un componente inercial y se ajusta a conductas esperadas en unas circunstancias dadas (Kahneman, 2003). En estas inercias conductuales existe una base cultural, pero igualmente moral que aporta un sentido al comportamiento propio y de los otros. Nuevamente, una ética de virtudes puede ayudar a comprender la dimensión moral de comportamiento económico que no surge de modo directo de normas, sino de valores aceptados y condicionada por factores contextuales (incertidumbre, poder, falibilidad...). 
El razonar socioeconómico trata de reconstruir un sólido concepto ético arraigado en una simple percepción: la cuestión de cómo abordar la escasez de recursos y bienes de un modo eficiente (económicamente racional) no puede estar separada de cómo se enfrenta a los conflictos sociales de un modo legitimado, es decir, justificado éticamente.

En este sentido, la propuesta planteada por P. Ulrich (1999) de una Economía Ética Integrativa, partiendo de una reflexión crítica sobre el modo de pensar económico, trata de penetrar en los supuestos normativos ocultos tras la lógica de mercado y sustituir el economicismo por la racionalidad socioeconómica. Los objetivos serían entonces, por un lado, la incorporación a la economía del amplísimo componente socio-cultural que subyace a la "generación de preferencias" y, por otro, la integración de la ética dentro de la lógica conductual de la propia economía. La adopción de este enfoque supone un notable cambio con respecto a las perspectivas funcionalista y correctiva, ya que, podría tratarse de un medio de acercar la Economía al complejo proceso de toma de decisiones realmente existente en el mercado (Koslowski, 1991).

\section{Conclusiones}

El problema del análisis ético de la economía tiene como punto de partida la deficiente integración de las dimensiones socioculturales en el estudio de la realidad económica. Las fuerzas del mercado son sociales en el más amplio sentido de la palabra y tras toda valoración económica subsiste una valoración moral.

El ser humano en cuanto ser social no puede establecer relaciones entre individuos sin adoptar decisiones morales, aun cuando buena parte de ellas sean realizadas tácita o "inconscientemente" en lo que denominamos disposiciones éticas o habitus. En definitiva, sin prestar atención a la dimensión moral no es abordable ninguna forma de relación social y, en concreto, las relaciones económicas también se construyen de normas sobre derechos, responsabilidades y comportamientos apropiados.

Un recorrido por los diversos intentos de integración pone en evidencia las deficiencias de conceptos tradicionales como enbedded, enmeshed o framed. La acción económica es una acción social determinada por instituciones sociales. Las deficiencias de integración del marco social en el análisis de hecho económico tiene también su traducción en la perspectiva que la ética asume en su análisis de la realidad económica y de la Economía en cuanto ciencia. Sintéticamente, podría distinguirse dos modos de comprensión de la relación existente entre ética y economía en razón de cuál de las dos asume el papel predominante: correctiva y funcionalista.

Una revisión del problema smithiano permite plantear una vía integrativa en la comprensión del papel de la ética en la realidad económica que supera las disociaciones de las propuestas precedentes. La ventaja de la alternativa smithiana reside en que su aproximación ofrece la posibilidad de comprender la moral económica de un modo en el que las consideraciones éticas no están escindidas realmente de las económicas. En concreto, la moral configura un sistema de precoordinación previo a la coordinación generada por las interacciones del mercado. Mediante la simpatía, la situación de los otros repercute en el proceso de toma de decisiones por parte del agente y sus acciones son juzgadas por un "espectador imparcial" constituido por un proceso de socialización.

Sistematizando las bases de una propuesta integrativa, ésta se apoya en dos pilares: la disolución de la dicotomía entre hechos y normas y el estudio de la racionalidad socioeconómica.

En definitiva, la ética económica no puede presentarse como un antídoto a la racionalidad económica, sino una reconstrucción comprehensiva de la racionalidad económica sobre bases antropológicas más realistas. La integración entre ética y economía implica una crítica de la concepción neoclásica divisora entre racionalidad y moralidad y la reconstrucción de los fundamentos éticos de la racionalidad socioeconómica. Lo que es lo mismo, una reorientación del razonar económico teniendo en cuenta la constitución ética de los individuos. Esto supone recuperar su dimensión crítica pero, igualmente, un acercamiento al proceso real de toma de decisiones en el que se entremezclan los aspectos culturales, éticos y económicos. En este punto la ética de virtudes plantea análisis más integrativo que las perspectivas deontológicas y consecuencialistas al uso. 
1 Entendemos por Economía Neoclásica a la orientación dominante 0 mainstream dentro de la Ciencia Económica. Sintéticamente, ésta se caracteriza por asumir los supuestos simplificadores sobre el comportamiento característicos de la Elección Racional, así como por la tendencia a la formalización matemática de sus propuestas. Al respecto puede verse (Screpanti y Zamagni, 1997).

2 Probablemente sólo es posible comprender el conjunto de los trabajos sobre este tema posicionándolos frente a esta aportación inicial. Así, las más recientes aportaciones continúan reelaborando el paradigma de esta obra para la que los hechos económicos se encuentran imbricados (embedment) en la realidad social (Smart, 2003 y Block, 2003).

3 La traducción a castellano resulta un tanto compleja. En su forma más directa sería integrado, e incluso incrustado. En nuestro caso optaremos por la primera opción. El término integración en castellano alude a una interconexión no conflictiva, matiz que no está presente en la obra de Polanyi. Probablemente el término enmeshing resultaba más clarificador, al aludir a una vinculación interna.

4 Con el término mainstream se alude a la "corriente principal" dentro de la Economía. En general podría considerarse como tal la Escuela Neoclásica en la medida en que esta representa el programa de investigación más general y más aceptado, hasta el punto de constituir la ortodoxia.

5 En general podemos hablar de al menos tres orientaciones en el estudio

Recibido: 21 de octubre de 2008 Aceptado: 26 de septiembre de 2011 leman o Becker, se centra en la aplicación del modelo neoclásico a problemas sociológicos (Becker, 1976), la Nueva Sociología Económica dan prioridad a la aplicación de una metodología sociológica a los problemas económicos (Granovetter, 1985). A su vez, la Socioeconomía aboga por introducir elementos propios de la psicología, sociología e incluso, ciencia política, para aproximar a la realidad a la mainstream (Etzioni, 1988).

6 En lo que respecta a la interacción entre redes sociales y economía, los estudios sobre "capital social" constituyen una de las principales líneas de investigación. Si bien, tanto los estudios sobre las redes sociales como el concepto de capital social tienen una larga tradición en sociología. El éxito de la obra de R. Putnam lo ha convertido en un tema central tanto en Sociología como en Economía (Putnam, 1991 y 1999). Puede verse un resumen del debate generado en (Sánchez y Pena, 2005).

7 Bajo el término OWERTY, esto es, la aparentemente irracional disposición de las letras en el teclado, a aquellas situaciones donde la evolución no parece haber conducido a resultados necesariamente más eficientes.

8 Este sería el caso de obras como la de Schweickart (1993) o de un sin fin de obras sobre moral económica planteadas desde la teología moral y por autores de orientación neomarxista, que proponen la construcción de un sistema económico alternativo.

9 Una sociedad tradicional que se encontrase en una situación de estancamiento o de equilibrio a bajo nivel, como de hecho podrian se consideradas las medievales, tenderia a realizar juicios éticos en los que se condenase cualquier forma de acumulación, planteando una ética alternativa a 
los resultados generados por el propio mercado.

10 Entre las obras de los autores que se encuentran en esta línea de pensamiento podemos citar las de (Novak, 1988; Bell, 1989; Buchanan 1994a y 1994b; Berger, 1991; Gilder, 1984; Fukuyama, 1995 y, por supuesto, Weber, 1994 [1905]).

11 De hecho, Etzioni recurre constantemente a la definición de dos funciones de utilidad que influyen en los comportamientos de los agentes: una individual y otra colectiva.

12 Dentro de las diversas alternativas en esta orientación merece la pena destacar la orientación de Steinmann, centrada en la defensa de una moral económica y empresarial asentada en una praxis dialógica. Se trata de una estrategia culturalista en la que partiendo de formas de vida compatibles pudiesemos lograr formas de organización y dirección más justas. Se trata en último término de desarrollar una ética apropiada para la creciente interculturalidad empresarial.

13 La denominación germana se ha convertido en la habitual del problema. De hecho los primeros en tematizar esa aparente contradicción fueron autores procedentes del ámbito académico alemán, concretamente, Lujo Brentano, Onken y Knies. Estos la atribuyeron al giro que habría supuesto para Smith el contacto con los pensadores liberales materialistas franceses y las idéas de la fisiocracia ("umschwungstheorie").

14 Este concepto ha sido fuertemente reivindicado por $A$. K. Sen, que en múltiples sentidos bebe de la tradición smithiana, para el que constituye una categoria esencial en el análisis de la incidencia de la cultura en la Economía. Al respecto puede verse (Sen, 1977, 1987).
15 Esta perspectiva de análisis es muy semejante a la adoptada por Benedetto Gui en su estudio de los bienes relacionales. Para este autor las relaciones sociales tienen un valor subjetivo no instrumental para sus participantes; (Gui, 2000).

16 El papel de la simpatía en los acuerdos económicos ha sido comprobado empíricamente tanto en el ámbito de la Economía Experimental como en el de la Behavioural Economics (Sally, 1995).

\section{BIBLIOGRAFÍA}

Anderson, E. S. (1993): Value in Ethics and Economics, Cambridge, Cambridge University Press.

Anspach, R. (1972): "The implications of the theory of moral sentiments for A. Smith's economic thought", History of political economy, 4, 176-206.

Becker, G. (1976): The economic approach to human behaviour, Chicago, University of Chicago Press.

Bell, D. (1989): Las contradicciones internas del capitalismo, Madrid, Alianza.

Berger, P. y Luckmann, T. (1966): The Social Construction of Reality: A Treatise in the Sociology of Knowledge, New York, Anchor Books.

Block, F. (2003): "Karl Polanyi and the writing of the great transformation", Theory and Society, 32, 275-306.

Buchanan, J. (1991): The economics and ethics of constitutional order, Ann Arbor, University of Michigan Press.

Buchanan, J. (1994a): Ethics and economic progress, Norman, University of Oklahoma press.

Buchanan, J. (1994b): Trust, the social virtues and the economic progress, London, Hamish Hamilton.

Buragoy, M. (1985): The Politics of Production: Factory Regimes Under Capitalism and Socialism, London, Verso.
Braudel, F. (1985): La dinámica del capitalismo, Madrid, Alianza Editorial.

Callon, M. (1998): The laws of market, Oxford, Blackwell.

Coase, R. (1937): "The Nature of the firm", Economica, New Series, 4 (16): 386405.

Conill, J. (2004): Horizontes de Economía Ética, Madrid, Tecnos.

Cortina, A. (1992): Ética de la empresa, Madrid, Trotta.

Cortina, A. y Conill, J. (2001): Educar en la ciudadanía, Valencia, Alfonso el Magnánimo.

Curtin, Ph. (1984): Cross-Cultural Trade in World History, Cambridge, Cambridge University Press.

Durkheim, E. ([1893] 1984): The division of labor in society, New York, The Free Press.

Etzioni, A. (1988): The moral dimension: towards a new economics, Nueva York, The Free Press.

Fukuyama, F. (1995): "El capital social y la economía mundial", Política exterior, 47, 89-102.

Gilder, G. (1984): Riqueza y pobreza, Madrid, Instituto de Estudios económicos.

Granovetter, M. (1985): "Economic action and social structure: the problem of embeddedness", The American Journal of Sociology, 91, 3, 481-510.

Gui, B. (2000): "Economics and interpersonal relations", Annals of public and cooperative economics, 71, 2, 133-138.

Habermas, J. (2000): Aclaraciones a la Ética del Discurso, Madrid, Trotta.

Hausman, D. y McPherson, M. (1993): "Taking ethics seriously", Journal of economics literature, 31, 2, 671-731.

Hausman, D. y McPherson, S. (2008): "The Philosophical foundation of mainstream normative economics", en Hausman, The Philosophy of economics, Cambridge, Cambridge University Press.

Hayek, F. (1994): Derecho, legislación y libertad, Madrid, Unión Editorial, 3. ${ }^{\text {a ed. }}$ 
Hempel, C. G. (1963): "Implications of Carnap's work for the philosophy of science", en P. Schilpp (ed.), The philosophy of R. Carnap, LaSalle, Opencourt, pp. 685-710.

Homan, K. y Pies, I. (1994): "Wirtshaftethik in der Moderne: Zur ökonomischen Theorie der Moral", Ethik und SociaIwissenshaften, 5, 3-12.

Hodgson, G. M. (1989): Economics and Institutions, Cambridge, Cambrigde Polity Press.

Jessop, B. (2000): "Regulationist and autopoieticist reflections on Polanyi's account of market economies and the market society", New Political Economy, 6, 2, 213-232.

Kahneman, D. (2003): "Maps of bounded rationality: psychology for behavioural economics", American Economic Review, 93, 5, pp. 1449-1475.

Koslowski, P. (1991): "Ethical economy as synthesis of economic and ethical theory", en P. Koslowski, Ethics in economics, business and economic policy, Berlin, Springer.

Koslowski, P. (1991): Ethics in economics, business and economic policy, Berlin, Springer.

Kripper, G. R. (2001): "The elusive market: embeddedness and the paradigm of economic sociology", Theory and society, 30, 775-810.

Luetge, C. (2005): "Economic ethics, business ethics and the idea of mutual advantages", Business ethics: a European review, 14, 2, 108-118.

Maclntyre, A. C. (1987): After virtue: a study on moral theory, London, Duckworth.

Martínez Real, F. J. (1998): "Entre la virtud y la eficiencia, teoría económica y ética", Estudios Filosóficos 136, 473-519.

Marwell, G. y Ames, R. (1981): "Economist free ride. Does anyone else?, experiments in the provision of public goods", Journal of public economics, 15, 295-310.
Mises, L. von (1994): Liberalismo, Barcelona, Planeta.

Mises, L. von (1995): La acción humana; tratado de economía, Madrid, Unión Editorial.

Nieli, R. (1986): "Spheres of intimacy and the A. Smith problem", Journal of the history of ideas, 47, 4, 611-624.

North, D. C. (1990): Institutions, institutional change and economic performance, Cambridge, Cambridge University Press.

Novak, M. (1988): El espíritu de capitalismo democrático, Buenos Aires, Tres tiempos, 5. ${ }^{\mathrm{a}}$ ed.

Nussbaum, M. (2001): Upheavals of Though: The Intelligence of Emotions, Cambridge, Cambridge University Press

Obershall, A. y Leifer, E. (1986): "Efficiency and Social Institutions: Uses and Misuses of Economic Reasoning in Sociology", Annual Review of Sociology 12: 233-253.

Okun, A. (1981): Prices and Quantities: $A$ Macroeconomic Analysis, Basil Blackwell, 0xford.

Pena López, J. A. y Sánchez Santos, J. M. (2007): "El problema de Smith y la relación entre Moral y Economía", Isegoría: Revista de filosofía moral y politica, 37, 81-103.

Polanyi, K. (1944): The Great Transformation, New York, Basic Books.

Putnam, H. (2002): The collapse of the Fact/ Value dichotomy, Cambridge, Harvard University Press.

Putnam, H. (2003): Ethics without ontology, Cambridge, Harvard University Press, 2003.

Putnam, H. (2003b): "For Ethics and economics without dichotomies" Review of political economy 15, 395-412.

Putnam, R. (1991): Making democracy work: civic traditions in modern Italy, Princeton, Princeton University Press.

Putnam, R. (1999): Bowling alone, the collapse and revival of American community, New York, Touchstone.
Quine, W. V. (1951): "Two dogmas of empiricism", Philosophical review, n. ${ }^{\circ} 60$, 20-43.

Sahlins, M ([1974] 1983): Economía de la Edad de Piedra. Madrid, Akal.

Sally, D. (1995): "Conversation and cooperation in social dilemmas: a meta-analysis of experiments from 1958 to 1992", Rationality and society 7, 58-92.

Sánchez Santos, J. M. y Pena López, J. A. (2005): "Actividad asociativa, confianza y generación de capital social: evidencia empírica", Ekonomiaz, 59, pp. 137-159.

Sayer, A. (2004): Moral Economy, Department of Sociology, Lancaster University.

Schweickart, D. (1993): Más allá del capitalismo, Santander, Salterrae.

Screpanti, E. y Zamagni, S. (1997): Panorama de la historia del pensamiento económico, Barcelona, Ariel.

Sen, A. K. (1967): "The nature and classes of precriptive judgements", The Philosophical Quarterly, 66, 46-62.

Sen, A. K. (1977): "Rational fools; a critique of the behavioural foundations of economic theory", Philosophy and public affairs, 6, 317-334.

Sen, A. K. (1987): Sobre ética y economía Madrid, Alianza.

Smart, B. (2003): Economy, culture and society, Buckingham, Open University Press.

Staveren, I. van (2007): "Beyond utilitarianism and deontology: ethics and economics", Review of Political Economy $19,1,21-35$.

Sugden, R. (1986): The economics of rights, cooperation and welfare, Blackwell, 0xford.

Titmuss, R. (1971): The gift relationship: from human blood to social policy, New York, Random House.

Ulrich, P. (1999): "Integrative Economic Ethics, towards a conception of socioeconomic rationality", en P. Koslowski, Contemporary Economic Ethics and Business Ethics, Berlin, Springer, pp. 37-55. 
Walsh, V. (2003): "Sen after Putnam", Review of political economy, 15, 3, pp. 315394.

Weber, M. (1994) [1905]: La ética protestante y el espiritu del capitalismo, Barcelona, Península.

West, E. G. (1976): Introduction to the TMS, Indianapolis, Liberty Press.

Williamson, E. O. (2000): "The New Institutional Economics, taking stock, looking ahead", Journal of Economic Literature, 38, 595-613.

Williamson, E. O. (1981): "The Economics of Organization: The Transaction Cost Approach", The American Journal of Sociology, 87(3), pp. 548-577.

Wilson, T. (1975): "Sympathy and selfinterest", en A. S. Skinner y T. Wilson, Essays on A. Smith, Oxford, Clarendon Press, pp. 73-111.
Young, J. T. (1997): Economics as a moral science, Cheltenham, E. Elgar.

Zamagni, S. (1989): "Sulla cooperazione tra scienza economica e etica: ragioni, problemi, prospettive", Orientamenti sociali, I, 65-77.

Zamagni, S. (1994): "On the ethical foundations of economic discourse", Economic notes, 2, 187-202. 